\title{
IEEE 802.11g PERFORMANCE IN PRESENCE OF BEACON CONTROL FRAMES
}

\author{
Elena Lopez-Aguilera, Jordi Casademont, Josep Cotrina
}

Wireless Networks Group, Telematics Department, Technical University of Catalonia (UPC)

\begin{abstract}
Most studies of the performance of IEEE 802.11 consider scenarios of ad-hoc topology networks and do not contemplate the network broadcast information contained in beacon frames. This paper presents a study of the performance of a WLAN whose infrastructure's topology is such that the access point is in charge of broadcasting the beacon frames. Thus, it is more realistic than previous studies, because beacon frames are usually transmitted in order to announce control information and network identity.

Furthermore, in the coverage area, user stations are likely to be working at different data rates, depending on their signal quality. Because beacon frames must be received by all stations, they are transmitted at the lowest data rate operating in the coverage area.

This article introduces a mathematical method to calculate the influence of beacon frames on the total throughput, collision probability and delays of the IEEE $802.11 \mathrm{~g}$ protocol. The model is validated by simulation analysis.
\end{abstract}

Index Terms- Beacon, IEEE 802.11, WLAN.

\section{INTRODUCTION}

- There have been many developments since 1997, when the Institute of Electrical and Electronics Engineers (IEEE) defined the first standard, IEEE 802.11, for wireless local area networks. IEEE 802.11 worked at $2.4 \mathrm{GHz}$ and at data rates of 1 and 2 Mbps. IEEE $802.11 \mathrm{~b}$, which at the same frequency achieved a data rate of $11 \mathrm{Mbps}$, appeared later. IEEE 802.1 la was developed subsequently; it reached 54 Mbps and its working frequency was increased to $5 \mathrm{GHz}$. This change of frequency, however, decreased its interoperability with older equipment. In answer to this, the IEEE $802.11 \mathrm{~g}$ was developed, which reaches $54 \mathrm{Mbps}$ but works at $2.4 \mathrm{GHz}$. In September 2003, a new working group began to develop IEEE $802.11 \mathrm{n}$, which should reach 100 Mbps. The working procedures are practically the same for all these standards; only the modulation, certain fields in the physical layer, the duration of the slot and the prioritising times (DIFS, SIFS and PIFS) change.

Among the other several IEEE 802.11 standards we should note that IEEE 802.11e defines procedures for managing network Quality of Service using classes of service.

Up to now, several papers have been written on various aspects of IEEE 802.11. [1] studies the throughput of the network considering radio coverage aspects and the hidden terminal problem. [2] and [3] show simulation and mathematical results of the throughput of an IEEE 802.11 single cell WLAN and also propose dynamic adjustments of the backoff algorithm to improve overall performance. In [4] and [5], we find several analyses of propagation issues. All these analyses are based on system traffic saturation and calculate the saturation throughput. More recently, several papers have appeared that work without this premise and consider situations of no congestion [6], [7]. Finally, the working group IEEE 802.11e that gives Quality of Service (QoS) possibilities to wireless LANs has also been studied in [8] and [9].

A common aspect in all these studies is that they do not consider the traffic caused by the broadcasting of network control information. Beacon frames announce the existence of a network and are an important part of many network maintenance tasks. They are transmitted at regular intervals to allow mobile stations to find and identify a network and to match parameters for joining the network. In an infrastructure network, the access point (AP) is responsible for transmitting beacon frames. The beacon period is not established by the standard and each Basic Service Set (BSS) can transmit beacon frames at its own specific interval. Usually, manufacturers set it at around $100 \mathrm{~ms}$, although the influence of this parameter on network throughput is yet to be determined.

Bearing these considerations in mind, the paper is organised as follows: Section II presents the main aspects of the IEEE 802.11 MAC working procedure, Section III describes the simulation environment, Section IV presents the mathematical model for determining the influence of beacon frames on network throughput, Section $\mathrm{V}$ validates the model through simulation and discusses several aspects of the presence of beacon frames, and Section VI summarises the most relevant points of the article.

\section{IEEE 802.11 MAC PROTOCOL}

IEEE 802.11 has two operating modes: Distributed Coordination Function (DCF) and Point Coordination Function (PCF). The most common working mode is DCF, which uses the medium access control (MAC) algorithm called CSMA/CA (Carrier Sense Medium Access with Collision Avoidance). It works as follows: before initiating a transmission, a station senses the channel to determine whether it is busy. If the medium is sensed idle during a period of time called Distributed Interframe Space (DIFS), the station is allowed to transmit. If the medium is sensed busy, the transmission is delayed until the channel becomes 
idle again. A slotted binary exponential backoff interval is uniformly chosen in $[0, \mathrm{CW}-1]$, where $\mathrm{CW}$ is the contention window. The backoff timer decreases as long as the channel is sensed idle, stops when a transmission is in progress, and is reactivated when the channel is sensed idle again for longer than the DIFS. When the backoff timer expires, the station attempts transmission. After each data frame is successfully received, the receiver transmits an acknowledgement frame (ACK) after a Short interframe Space (SIFS) period. The value of CW is set to 32 in the first transmission attempt, and ascends in integer powers of 2 at each retransmission, up to a pre-determined value (usually 1024).

Beacon frames serve several fundamental purposes in an infrastructure network. At the most basic level, beacon frames define the coverage area of a BSS. User stations monitor beacon frames to determine which Extended Service Set (ESS) offers coverage at their physical location and they use the received signal strength to monitor signal quality. They also broadcast information on the buffered data frames of the saving power function. In infrastructure networks, beacon frames are transmitted periodically by the AP. In ad-hoc networks, a distributed procedure coordinates the transmission of beacon frames between user stations. Beacon intervals can be configured by the AP manager; the shorter the beacon period, the more traffic generated.

Fig. 6 shows all the fields that can be used in a beacon frame in the order in which they appear. Not all of the elements are present in all beacons. Optional fields are present only when there is a reason for them to be used. The FH Parameter Set is used only when the underlying physical layer is based on frequency hopping. The DS Parameter Set is employed when the user stations use direct-sequence techniques or extended rate PHY (ERP). Only one physical layer can be in use at any point, so the FH and DS Parameter Sets are mutually exclusive.

The CF (Contention Free) Parameter Set is used only in frames generated by access points that support the PCF, which is optional. The TIM (Traffic Indication Map) element is used only in beacons generated by access points when the saving power function is activated and the AP performs frame buffering.

Therefore, because the beacon packet length is variable, we took regular beacons that were 106 bytes in length, including the MAC-Header. We assumed all the stations used Direct Sequence Spread Spectrum (DSSS) or the ERP physical layer, so we included the DS Parameter Set field and not the correspondent $\mathrm{FH}$ field. We took into account the fact that the access point supports only the DCF operating mode and not the PCF, so we did not include the CF Parameter Set. In addition, we considered the saving power function of the access point to be inactive; therefore, we did not include the TIM fields in the beacon frame body.

\section{SIMULATION ENVIRONMENT DESCRIPTION}

In order to analyse the performance of the IEEE $802.11 \mathrm{~g}$, we used a simulation tool implemented at UPC. The simulation program, written in $\mathrm{C}++$, follows the IEEE 802.11 protocol and permits the evaluation of different parameters: throughput (user data correctly transmitted by users without considering retransmissions and headers), average transmission delay, average queue delay, time period during which all network stations are in backoff state, and probability of collision. The simulation tool was verified by comparing the results obtained with the results published in [2], which were obtained under identical simulation conditions.

The simulation environment consists of 1 BSS composed of $1 \mathrm{AP}$ and 10 user stations. Only user stations transmitted data packets with a constant payload size of 1023 bytes. We consider the data to be directed from user stations towards the AP, who forwards them to the infrastructure network. All the user stations were within the coverage area. Hidden terminal situation and transmission errors were not considered.

Table I. Main parameters used in the simulations

\begin{tabular}{|c|c|c|}
\hline & $\begin{array}{c}802.11 \mathrm{~g} \\
(802.11 \mathrm{~b}- \\
\text { compatible })\end{array}$ & $\begin{array}{c}802.11 \mathrm{~g} \\
\text { (ERP-OFDM) }\end{array}$ \\
\hline Transmission data rate (Mbps) & $1,2,5.5,11$ & $\begin{array}{c}6,9,12,18 \\
24,36,48,54\end{array}$ \\
\hline MAC hcader & \multicolumn{2}{|c|}{34 bytes } \\
\hline $\mathrm{ACK}$ & \multicolumn{2}{|c|}{14 bytes } \\
\hline Propagation time & \multicolumn{2}{|c|}{$1 \mu \mathrm{s}$} \\
\hline Long PHY preamble & $144 \mu \mathrm{s}$ & \multirow{2}{*}{$16 \mu \mathrm{s}$} \\
\hline Short PHY preamble & $72 \mu \mathrm{s}$ & \\
\hline Long PHY header & $48 \mu \mathrm{s}$ & \multirow{2}{*}{$4 \mu \mathrm{s}$} \\
\hline Short PHY header & $24 \mu \mathrm{s}$ & \\
\hline Slot time & \multicolumn{2}{|c|}{$20 \mu \mathrm{s}$} \\
\hline SIFS & \multicolumn{2}{|c|}{$10 \mu \mathrm{s}$} \\
\hline DIFS & \multicolumn{2}{|c|}{$50 \mu \mathrm{s}$} \\
\hline PIFS & \multicolumn{2}{|c|}{$30 \mu \mathrm{s}$} \\
\hline Minimum backoff window size & \multicolumn{2}{|c|}{32} \\
\hline Maximum backoff window size & \multicolumn{2}{|c|}{1024} \\
\hline Beacon length & \multicolumn{2}{|c|}{106 bytes } \\
\hline
\end{tabular}




\section{MATHEMATICAL MODEL FOR DETERMINING THE INFLUENCE OF BEACON FRAMES ON THROUGHPUT}

The proposed scenario considers an infrastructure BSS in which the AP sends beacons and $n$ user stations continually send data packets with a constant payload. Transmission errors are not considered.

We consider that, if the AP has to transmit a beacon, and the channel is sensed to be busy, the AP will wait until the channel remains idle for a PCF Interframe Space (PIFS) period, where DIFS $>$ PIFS $>$ SIFS. Otherwise, if the channel is sensed to be idle, the AP will transmit the beacon frame immediately.

In order to evaluate the influence of the beacon traffic, we used Bianchi's model [2] as a starting point. This model concludes with the following expression for the saturation throughput $S$ :

$$
S=\frac{P_{t r} P_{S} E[P]}{E[\text { Slot }]}
$$

where $E[S l o t]$ is the average length of the backoff time slot, $E[P]$ is the average length of a packet payload, Ptr is the probability that at least one user station is transmitting and $P_{S}$ is the probability that a transmission will be successful. E[Slot] can be calculated by

$$
E[S l o t]=(1-P t r) \sigma+\operatorname{PtrPs} T s+\operatorname{Ptr}(1-P s) T c
$$

where $\sigma$ is the duration of an empty slot, $T s$ is the time length of a successful transmission and $T c$ is the time the channel is sensed busy during a collision.

When evaluating the influence of beacon traffic, note that the probability of a collision occurring between a transmitted data packet and a beacon packet may be omitted, because it is very low.

Bianchi's model remains applicable even when beacons are transmitted. Note that only the average slot time E[slot] will be modified, because new slots are produced by the presence of beacons.

Now, considering a beacon period of $1 / \lambda \mu s$, we need to compute the extent to which beacon transmissions prolong the channel busy period.

When a beacon arrives, the probability that a channel will be busy is

$$
P_{o}=\frac{(P \operatorname{tr} P s(T s-D I F S)+P \operatorname{tr}(1-P s)(T c-D I F S))}{E[\operatorname{slot}]}
$$

In this case, the beacon is transmitted PIFS $\mu$ s after the channel is sensed idle. After the transmission, all stations wait during a $D I F S$ period. In this case, the DIFS period is omitted because of the beacon transmission. Therefore, the beacon transmission prolongs the busy period by

$$
T_{b o}=\text { PHYPreamble }+ \text { PHYHeader }+E[\text { beacon }]+\delta+P I F S
$$

where E[beacon] is the beacon length time and $\delta$ is the propagation time.

If the channel is sensed idle when the beacon arrives, two cases may arise. The first case is when a beacon arrives during a DIFS period. The probability of this occurring is

$$
P_{i 1}=\text { PtrDIFS } / E[\text { slot }]
$$

and the beacon transmission prolongs the average busy period by

$T_{b i 1}=$ PHYPreamble + PHYHeader + E[beacon $]+\delta+\frac{1}{2}$ DIFS

The second case occurs when a beacon arrives during an empty slot. The probability of this happening is

$$
P_{i 2}=(1-P t r) \sigma / E[s l o t]
$$

The beacon transmission prolongs the busy period by

$T_{b i 2}=$ PHYPreamble + PHYHeader $+E[$ beacon $]+\delta+D I F S$

Thus, on average, beacon transmission prolongs the busy period by

$$
T b=P_{o} T_{b o}+P_{i 1} T_{b i 1}+P_{i 2} T_{b i 2}
$$

Therefore, the new average slot time is

$$
E^{\prime}[\text { slot }]=\frac{1 / \lambda}{1 / \lambda-T b} E[\text { slot }]
$$

Finally, when beacons are transmitted, the saturation throughput $S^{\prime}$ is

$$
S^{\prime}=\frac{E[\text { slot }]}{E^{\prime}[\text { slot }]} S=\frac{1 / \lambda-T b}{1 / \lambda} S
$$

Using Bianchi's model, the average transmission delay is computed as

$$
T t=\frac{E[s l o t]}{\tau(1-\tau)^{n-1}}
$$

Therefore, when beacons are transmitted, the new average transmission delay is

$$
T t^{\prime}=\frac{E^{\prime}[\text { slot }]}{\tau(1-\tau)^{n-1}}=T t \frac{E^{\prime}[\text { slot }]}{E[\text { slot }]}=\frac{1 / \lambda}{1 / \lambda-T b} T t
$$

\section{MODEL VALIDATION AND SYSTEM BEHAVIOR}

To validate the model, we compared the mathematical results with those obtained with the simulation tool presented in the previous section. The fact that the analytical results (lines) coincide with the simulation results (symbols) shows that the analytical model is accurate.

We must take into account that IEEE 802.11g uses different physical layers depending on the data rates used:

- For 1, 2, 5.5 and $11 \mathrm{Mbps}$, it uses the 802.11b physical layer. For $1 \mathrm{Mbps}$, it uses the long PHY format. For $2,5.5$ and $11 \mathrm{Mbps}$, it is possible to choose between the long and short PHY formats.

- For higher rates $(6,9,12,18,24,36,48$ and 54 Mbps), it uses the ERP-OFDM physical layer. For all these data rates, it is possible to use either the long slot time (20 $\mu \mathrm{s})$ or the short slot time (9 $\mu \mathrm{s})$. 
User stations present in the system employ different physical layers, depending on the data rates they use. The use of the long slot time is thus mandatory for user stations employing ERP-OFDM, if any of the stations in the coverage area uses the IEEE $802.11 \mathrm{~b}$ physical layer. In this way, compatibility between the two layers is provided.

Wireless networks are evolving towards higher transmission data rates, but these high rates are only useful when the distance between the transmitter and the receiver is short or when the signal transmission is of high quality. When a user station is placed near the limit of the coverage area or the transmission between the transmitter and the receiver is of low quality due to bad propagation conditions, the operating data rate has to be reduced to a lower speed. Because the beacon frames must be received by all the stations in the BSS, they are transmitted at the lowest data rate operating in the coverage area.

Fig. 1 shows the normalised saturation throughput versus beacon periods, considering different transmission rates. We are able to distinguish two cases:

- The beacon frames transmission occurs at the same rate as the data packets.

- The beacon frames are transmitted at the lowest possible data rate, $1 \mathrm{Mbps}$.

If beacon and data frames are transmitted at the same rate, the presence of beacons does not influence network performance at high data rates (more than $6 \mathrm{Mbps}$ ). On the other hand, if beacon frames are transmitted at $1 \mathrm{Mbps}$, due to the presence in the BSS of user stations operating at this low data rate, then the figure shows that beacon traffic reduces saturation throughput up to a beacon period of 100 $\mathrm{ms}$. At a beacon period of $25 \mathrm{~ms}$, this decrease takes a value of about $4 \%$, for the various data rates.

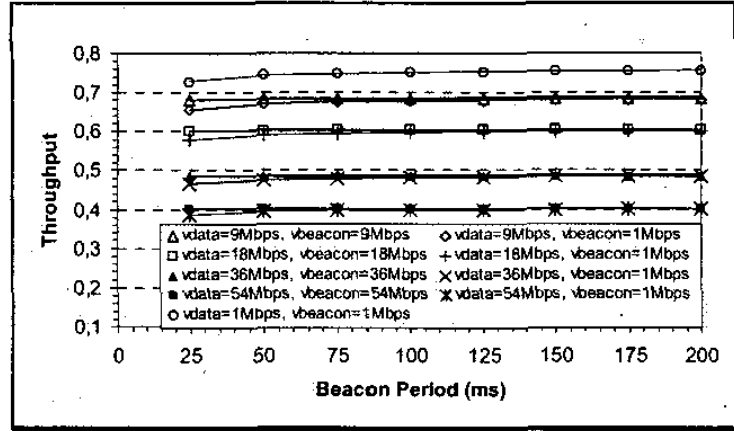

Fig. 1. Saturation throughput versus beacon period at various beacon and data transmission rates

The data packet's collision probability (Fig. 2) is not influenced by variations in the beacon period. As previously stated, beacon frames take priority over data packets and thus they rarely collide; consequently, the number of collisions per transmitted packet does not increase or decrease. Moreover, the collision probability remains the same for different data and beacon rates. In this way, the probability that a data packet transmitted will collide depends on the values of the interframe space times (DIFS, SIFS, PIFS) that control the medium access and that remain unchanged for the different data rates employed.

Moreover, we studied the beacon packets' influence on the percentage of time that the network remains idle in a backoff procedure (Fig. 3). If beacon and data frames are transmitted at the same rate, the presence of beacons does not influence backoff time for high data rates. If beacon frames are transmitted at $1 \mathrm{Mbps}$, however, the period of time during which the medium is busy due to a beacon packet transmission grows and, in this way, the percentage of backoff time decreases slightly up to a beacon period of $100 \mathrm{~ms}$.

Another parameter that may be relevant to study is the transmission delay. This is the interval of time that starts at the instant when a packet is ready to be transmitted (that is, when it is the first one in its transmission queue) and finishes when the corresponding $\mathrm{ACK}$ packet is received. Fig. 4 shows transmission delay as a function of the beacon period. As with the parameters studied until now, if beacon and data frames are transmitted at the same rate, the presence of beacons does not influence transmission delay at high data rates. On the contrary, if beacon frames are transmitted at $1 \mathrm{Mbps}$, beacon traffic increases transmission delay up to a beacon period of $100 \mathrm{~ms}$. At a beacon period of $25 \mathrm{~ms}$, this growth takes a value of about $4 \%$, for the various data rates.

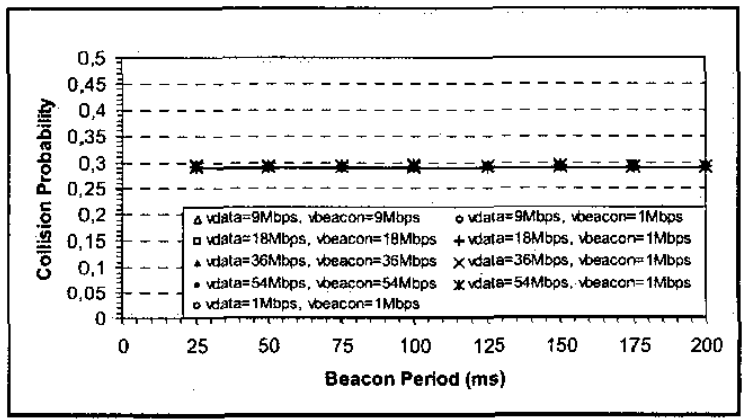

Fig. 2. Collision probability versus beacon period at various beacon and data transmission rates

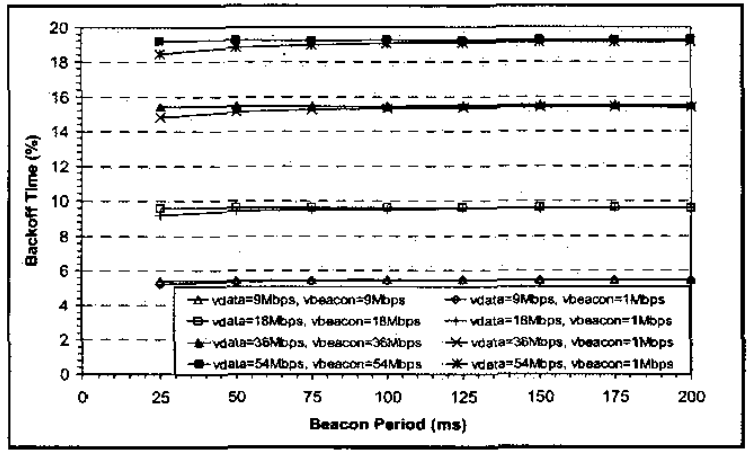

Fig. 3. Backoff time versus beacon period at various beacon and data transmission rates

Fig. 5 shows another approach to saturation throughput, in which beacon packets are transmitted at $1 \mathrm{Mbps}$. In this case, various payload lengths, beacon periods and data rates 
are used. Between a beacon period of $25 \mathrm{~ms}$ and another of $100 \mathrm{~ms}$, the throughput difference takes a value of about $3.25 \%$, for the various data rates. Moreover, as we already know from previous studies, saturation throughput decreases with the length of data packets; this behaviour is the same for different beacon periods. The differences in throughput for different payload sizes are constant for different beacon periods.

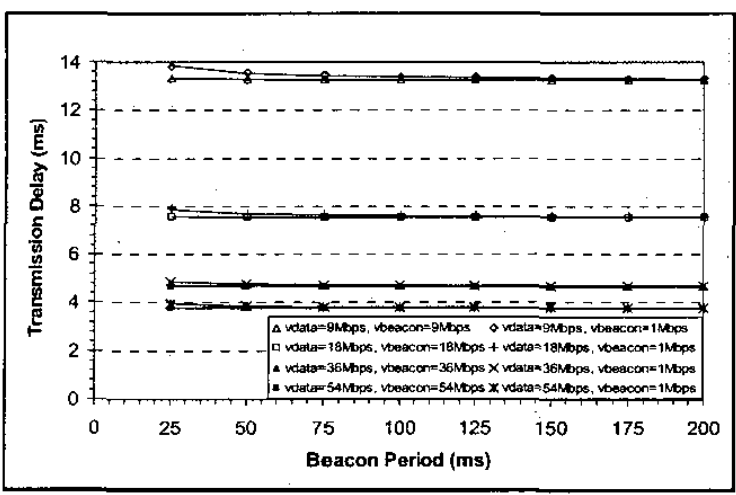

Fig. 4. Transmission delay versus beacon period at various beacon and data transmission rates

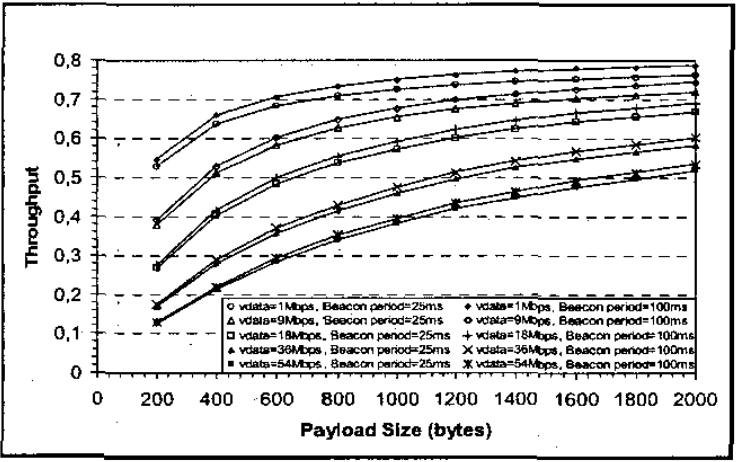

Fig. 5. Saturation throughput versus payload size at various beacon periods and data transmission rates

\section{CONCLUSIONS}

This paper presents a study of the influence of beacon frames on the performance of IEEE $802.11 \mathrm{~g}$ networks. In the coverage area, user stations will be operating at different rates. Beacon frames have to be received by all stations in the BSS; therefore, they are transmitted at the lowest data rate in the coverage area. We studied the influence of beacon frames transmitted at $1 \mathrm{Mbps}$ on network performance.

In order to evaluate this influence, a mathematical model is presented, which was validated through software simulation. Although the results show that beacon frames do not have much of an influence on network performance, it is certainly worth documenting this fact and obtaining a quantifying number to demonstrate it objectively.

\section{ACKNOWLEDGMENTS}

This work was partly funded by the CICYT project TIC2003-01748.

\section{REFERENCES}

[1] H. S. Chaya, S. Gupta, "Performance modeling of asynchronous data transfer methods of IEEE 802.11 MAC protocol", Wireless Networks, Kluwer Academic Publishers, Vol. 3, No. 3, 1997, pp. 217 - 234.

[2] G. Bianchi, "Performance analysis of the IEEE 802.11 Distributed Coordination Function", IEEE Journal on selected areas in communications, Vol. 18, No. 3, March 2000, pp. 535 547.

[3] F. Cali, M. Conti, E. Gregori, "Dynamic tuning of the IEEE 802.11 protocol to achieve a theoretical throughput limit", IEEE /ACM Transactions on networking, Vol. 8, No. 6, December 2000 , pp. 785 - 799

[4] Z. Hadzi-Velkov, B. Spasenovski, "On the capacity of IEEE 802.11 DCF with capture in multipath-faded channels", International journal of wireless information networks, Kluwer Academic Publishers, Vol. 9, No. 3, July 2002, pp. 191 - 198.

[5] M. V. Clark, K. K. Leung, B. McNair, Z. Kostic, "Outdoor IEEE 802.11 cellular networks: radio link performance", IEEE International Conference on Communications 2002 (ICC 2002), Vol. 1, April 2002, pp. $512-516$.

[6] E. Ziouva, T. Antonakopoulos, "The IEEE 802.11 Distributed Coordination Function in small-scale ad-hoc Wireless LANs", International journal of wireless information networks, Kluwer Academic Publishers, Vol. 10, No. 1, January 2003, pp. 1 - 15.

[7] O. Tickoo, B. Sikdar, "On the impact of IEEE 802.11 MAC on traffic characteristics", IEEE Journal on selected areas in communications, Vol. 21, No. 2, February 2003, pp. 189 - 203.

[8] I. Aad, C. Castelluccia, "Differentiation mechanisms for IEEE 802.11", INFOCOM 2001, Vol. 1, April 2001, pp. 209 - 218.

[9] S. Mangold, S. Choi, P May, O. Klein, G. Hiertz, L. Stibor, "IEEE 802.11 e Wireless LAN for Quality of Service", Proc. European Wireless (EW'2002), Vol. 1, February 2002, pp. 32-39.

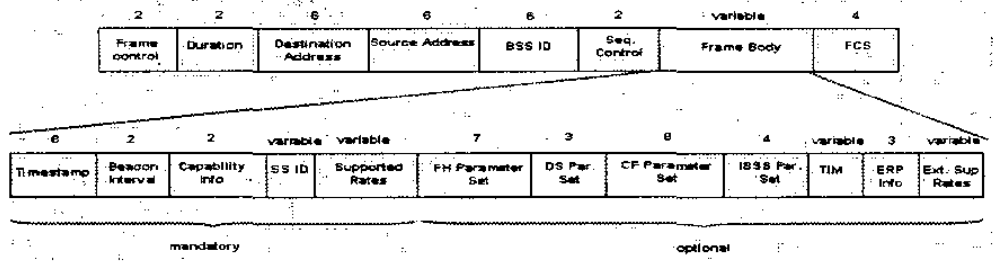

Fig. 6. Beacon Frame 